\title{
Lef1 plays a role in patterning the mesoderm and ectoderm in Xenopus tropicalis
}

\author{
GIULIETTA ROËL ${ }^{1}$, YOONY Y.J. GENT ${ }^{3}$, JOSI PETERSON-MADURO², FONS J. VERBEEK ${ }^{4}$ \\ and OLIVIER DESTRÉE*,2 \\ ${ }^{1}$ Max-Delbrück Center for Molecular Medicine, Berlin, Germany, ${ }^{2}$ Hubrecht Institute, Utrecht, ${ }^{3}$ Department of Dermatology and Allergol- \\ ogy, UMC, Utrecht and ${ }^{4}$ LIACS, Leiden University, Leiden, The Netherlands
}

\begin{abstract}
Tcf/Lef HMG box transcription factors are nuclear effectors of the canonical Wnt signaling pathway, which function in cell fate specification. Lef1 is required for the development of tissues and organs that depend on epithelial mesenchymal interactions. Here, we report the effects of lef1 loss of function on early development in $X$. tropicalis. Depletion of lef1 affects gene expression already during gastrulation and results in abnormal differentiation of cells derived from ectoderm and mesoderm. At tail bud stages, the epidermis was devoid of ciliated cells and derivatives of the neural crest, e.g. melanocytes and cephalic ganglia were absent. In the Central Nervous System, nerve fibers were absent or underdeveloped. The development of the paraxial mesoderm was affected; intersomitic boundaries were not distinct and development of the hypaxial musculature was impaired. The development of the pronephros and pronephric ducts was disturbed. Most striking was the absence of blood flow in lef1 depleted embryos. Analysis of blood vessel marker genes demonstrated that lef1 is required for the development of the major blood vessels and the heart.
\end{abstract}

KEY WORDS: lef1, Xenopus, tropicalis, knockdown, wnt

\section{Introduction}

The Tcf/Lef family of HMG box transcription factors functions in the development of multiple tissues and organs during development as well as in the maintenance of stem cell compartments in adult tissues. The vertebrate Tcf/Lef family consists of four members: Tcf1 (TCF7), Lef1 (LEF1), Tcf3 (TCF7L1) and Tcf4 (TCF7L2) (reviewed in Arce et al., 2006). The interaction of Tcf/ Lef proteins with $\beta$-catenin is required for the formation of a transcriptional active protein complex to regulate target gene expression (references in Arce et al., 2006). Tcf/Lef proteins also function as transcriptional repressors by binding to members of the Groucho/TLE family. Groucho related proteins mediate a repressor function for all Tcf/Lef proteins through interaction with histone-deacetylase-1 (references in Arce et al., 2006). Lef1 is required for the proper development of hair follicles, mammary glands and teeth which all depend on epithelial mesenchymal interactions (van Genderen et al., 1994). Lef1 deficient mice lack the mesencephalic nucleus of the trigeminal nerve, which is the only neural crest-derived neuronal population affected (van Genderen et al., 1994).
The endogenous expression pattern of Lef1 in the mouse and Xenopusindicates additional functions also during early development (Oosterwegel et al., 1993; Molenaar et al., 1998; Galceran et al., 1999). Xenopus lef1 is expressed at high levels in the branchial arches and neural crest derived cells, in the developing heart, lateral plate mesoderm, the tail bud, fins and the mesencephalon (Molenaar et al., 1998). In addition, Lef1 and Tcf1 double mutant mice showed additional developmental abnormalities compared to the single mutants and demonstrated redundant functions between Lef1 and Tcf1 during development (Galceran et al., 1999).

In Xenopus, lef1 expression starts at stage 9 (Molenaar et al., 1998) just prior to mesoderm specification and successive patterning of the mesoderm during gastrulation. Mesoderm patterning is under the control of different signaling pathways including

\footnotetext{
Abbreviations used in this paper:APJ, angiotensin Ireceptor-like 1; Lef, lymphoid enhancer-binding factor; Msr, mesenchyme-associated serpentine receptor/ angiotensin receptor related protein; Myf, myogenic factor; nr-3, nodalrelated-3; Wnt, wingless-type MMTV integration site family.
}

\footnotetext{
*Address correspondence to: Dr. O. Destrée. Hubrecht Institute, Uppsalalaan 8, 3584 CT, Utrecht, The Netherlands. Fax: +031-30-2516-464. e-mail: o.destree@niob.knaw.nl - http://www.niob.knaw.nl
} 
Wnt signaling. In Xenopus, wnt8 is required to pattern the ventrolateral mesoderm (Christian and Moon, 1993; Hoppler et al., 1996) and restricts the dorsal mesoderm to the prospective dorsal side (Hoppler and Moon, 1998). Recently, we showed in loss of function experiments that lef 1 is downstream of wnt8 and is required for patterning the paraxial mesoderm by regulating the expression of myod(Roël et al., 2002).

Here, we report the effects of loss of function of lef 1 during development in Xenopus tropicalis. We show that lef1 is differentially expressed already at gastrula stages in the ectoderm and the mesoderm. Loss of function experiments demonstrate that lef1 is required for the proper expression of nodal related 3, myod and $m s r / A P J$ to pattern the dorsal and ventro-lateral mesoderm during gastrulation. Our results indicate that in Xenopus lef1 regulates the genetic program of somitogenesis through myod but not through myf5. Analysis of lef1 depleted embryos at the tail bud stage revealed impaired development of different tissues and organs derived from the ectoderm, the mesoderm and the endoderm. In particular, formation of major blood vessels and the pronephric ducts that are derived from the lateral plate mesoderm (Walmsley et al., 2002) was affected. The different tissue components of the heart, i.e. endocardium and myocardium, did not develop properly.

\section{Results}

\section{Xenopus lef1 lacks exon $\mathrm{VI}$}

Xenopus lef1 cDNA sequences reported so far (Molenaar et al., 1998) do not contain the exon, annotated as VI in human $\angle E F 1$, which is also represented in other Tcf/Lef members (Arce et al., 2006). LEF1 exon VI encodes an activation domain and exon $\mathrm{VI}$ isoforms may have different functions during development (Gradl et al., 2002). Analysis of Xenopus genomic lef1 sequences between corresponding exons $\mathrm{V}$ and $\mathrm{VII}$ revealed that lef1 does not contain exon VI related sequences (data not shown). Also, sequence analysis of lef1 of Fugu rubripes, using the genomic database of the pufferfish (DOE Joint Genome Institute), revealed the lack of exon VI related sequences. A more extensive phylogenetic analysis is required to determine whether this exon was acquired in mammalian genomes or lost in amphibians and fish. We have shown by northern-blot analysis that lef1 produces only one mRNA during early Xenopus development (Molenaar et al., 1998). In addition, screening of XenopuscDNA libraries and 5'-RACE did not uncover evidence for alternative splicing of lef1 (data not shown). Thus, our lef1 knockdown experiments in Xenopus concern the loss of function of a single lef1 isoform not containing the exon VI encoded activation domain present in mammals.

\section{Lef1 is required for patterning the mesoderm in Xenopus tropicalis}

To determine functions of lef1 during early development we analyzed the effects of knockdown with antisense morpholino oligonucleotides in $X$. tropicalis embryos. $X$. tropicalis has a diploid genome and is therefore a favorite organism for gene knockdown experiments (Nutt et al., 2001). We determined the endogenous expression pattern of lef1 in $X$. tropicalis with $X$. tropicalis specific probes (Fig. 1A and Roël et al., unpublished results), which appeared to be very similar to the previously described expression pattern in X. laevis (Molenaar et al., 1998). Expression of lef1 starts at Mid-Blastula Transition, i.e. before gastrulation (Molenaar et al., 1998). Lef1 is expressed at high levels in lateral and ventral domains of the involuting mesoderm overlapping with the wnt8expression domain (Fig. 1B and Christian and Moon, 1993), and it is also expressed in the adjacent endoderm (Fig. 1A).

To block lef1 protein expression during early development, embryos were injected at the two-cell stage with $10 \mathrm{ng}$ of lef1 morpholino antisense oligonucleotide (morpholino) in each blastomere. In rescue experiments, embryos were co-injected with lef1 morpholino and human $\angle E F 1$ RNA, which is not sensitive to the morpholino.

Analysis of gastrula stage embryos showed that lef1 depletion

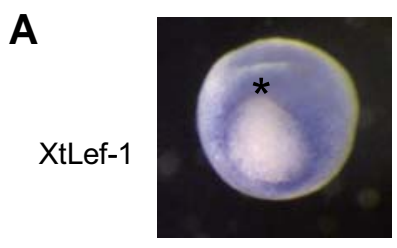

B Control Lef-1 MO Lef-1 MO + hLEF RNA

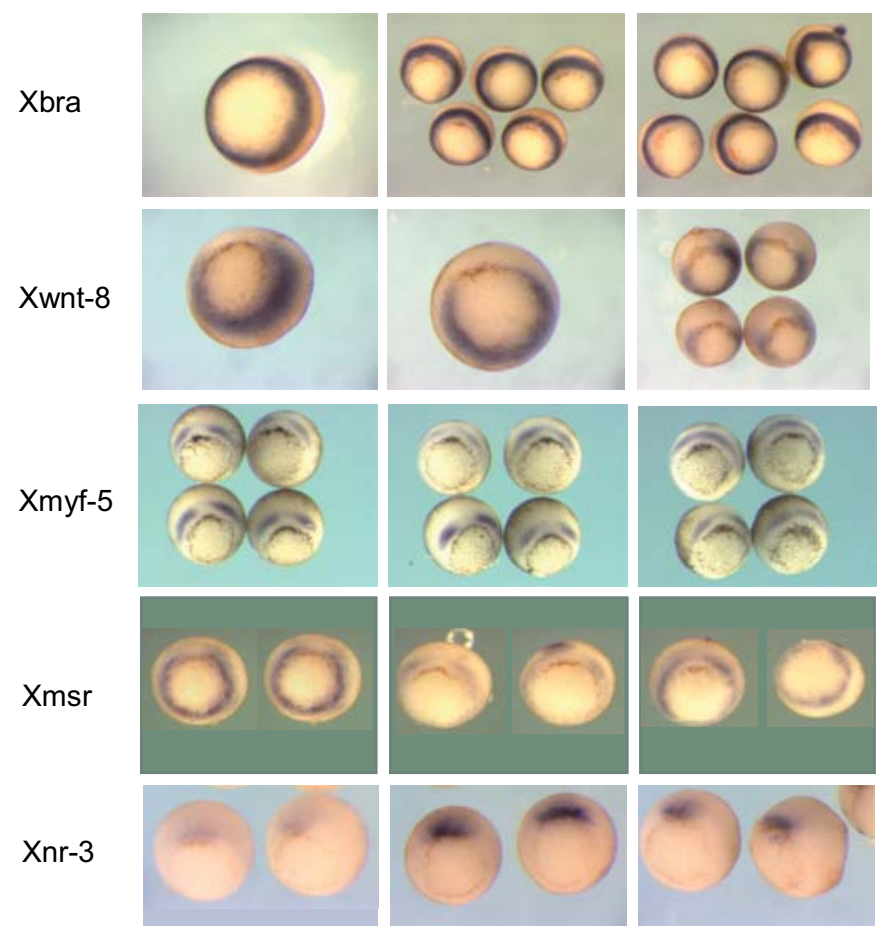

Fig. 1. Lef1 depletion affects expression of genes in the ventral and dorsal mesoderm. In situ hybridization for $\mathrm{X}$. tropicalis lef1 RNA at stage 10.5 (A) shows high levels of expression in the lateral and ventral marginal zone, low expression in the ectoderm and in the dorsal marginal zone (dorsal side up). Expression of marker genes in gastrula stage embryos, stage 10.5 (B), dorsal side up. Lef1 depletion does not alter the expression of brachyury, wnt8 or myf5. Msr/APJ expression is downregulated by lef1 depletion whereas nodal related $3(\mathrm{nr}-3)$ expression is upregulated. Co-injection of human LEF1 RNA rescues the expression of msr/APJ and partially downregulates nr-3 expresssion. 
did not affect the expression of the pan-mesodermal marker gene brachyury (Fig. 1B), indicating that lef1 is not required for mesoderm specification. Both Myodand Myf5 were found to be Wnt targets in mouse paraxial mesoderm explants (Tajbakhsh et al., 1998) and, in Xenopus, myodand myf5 are expressed within the lef1 expression domain. Promoter analysis showed that $m y f 5$ expression might be regulated by $\beta$-catenin/tcf in Xenopus (Yang et al., 2002; Shi et al., 2002). However, the expression of myf5 at mid-gastrula stage was not affected by lef1 depletion (Fig. 1B) in contrast to that of myod (Roël et al., 2002). These results demonstrate that, in Xenopus, lef1 is required for activation of only part of the myogenic genetic program to pattern the presomitic mesoderm during gastrulation.

The expression domain of the endothelial marker $\mathrm{msr}$ (Devic et al., 1996) in the gastrula embryo overlaps with the expression domains of lef1 and wnt8 (Christian and Moon, 1993) and contains high levels of nuclear $\beta$ catenin (Schohl and Fagotto, 2002). Therefore, msrmay be regulated by Wnt/ $\beta$-catenin/lef1. Indeed, lef1 depletion resulted in down regulation of msrexpression in the marginal zone in all of the embryos analysed by in situ hybridisation ( $n=14)$ (Fig. 1B). Wnt8 expression was not affected by lef1 depletion (Fig. 1B) indicating that these effects of lef1 depletion were not due to an indirect effect via wnt8. Co-expression of mRNA encoding human $\angle E F 1$ restored the expression of $M s r / A P J$ in all of the embryos analysed ( $n=12)$ (Fig. 1B). These results demonstrate that lef1 is required for normal expression of $m s r / A P J$ in the marginal zone and suggest that $m s r / A P J$ may be a new Wnt response gene downstream of lef1 signaling.

Since lef1 is also expressed in the dorsal marginal zone (Fig. 1A) we asked whether lef1 also functions in patterning the organizer of the Xenopus embryo. Therefore, we analyzed the expression of nodal related 3 , which is a direct target of maternal $\beta$-catenin/tcf3 (McKendry et al., 1997; Houston et al., 2002). To our surprise, expression of nodal related 3 was elevated at the endogenous site with an ectopic extension towards the animal pole (Fig. 1B), as after depletion of maternal tcf3 (Houston et al., 2002). These results show that lef 1 is required for the proper expression of nodal related 3 in the dorsal marginal zone.

\section{Phenotypic analysis of lef1 depleted embryos at later stages}

We studied the effects of lef1 depletion on later development in more detail. During tail bud stages, growth of $74 \%(n=178 / 241)$ of the lef 1 depleted embryos was strongly retarded, resulting in embryos with a very short tail (Fig. 2B). Lef1 depleted embryos lacked both dorsal and ventral fins and pigmentation of neural crest derived melanophores. The head and eyes were smaller and the eyes were less pigmented compared to those of noninjected controls (Fig. 2A,B). Moreover, lef1 depleted embryos did not show a blood flow and did not respond to mechanical stimuli.

Histological analysis and 3D-reconstructions of representative lef1 depleted embryos show that many of the major organs are present at stage 38, including brain and spinal cord, notochord,
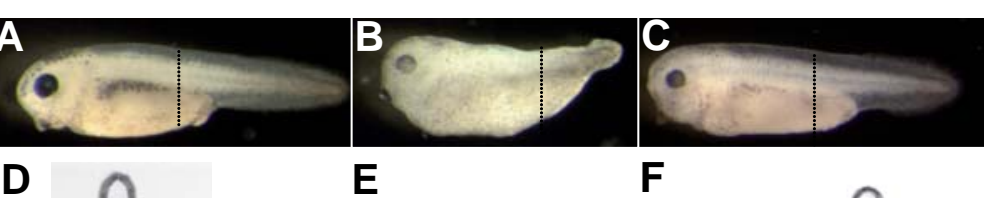

$\mathbf{F}$

dorsal fin

dlav
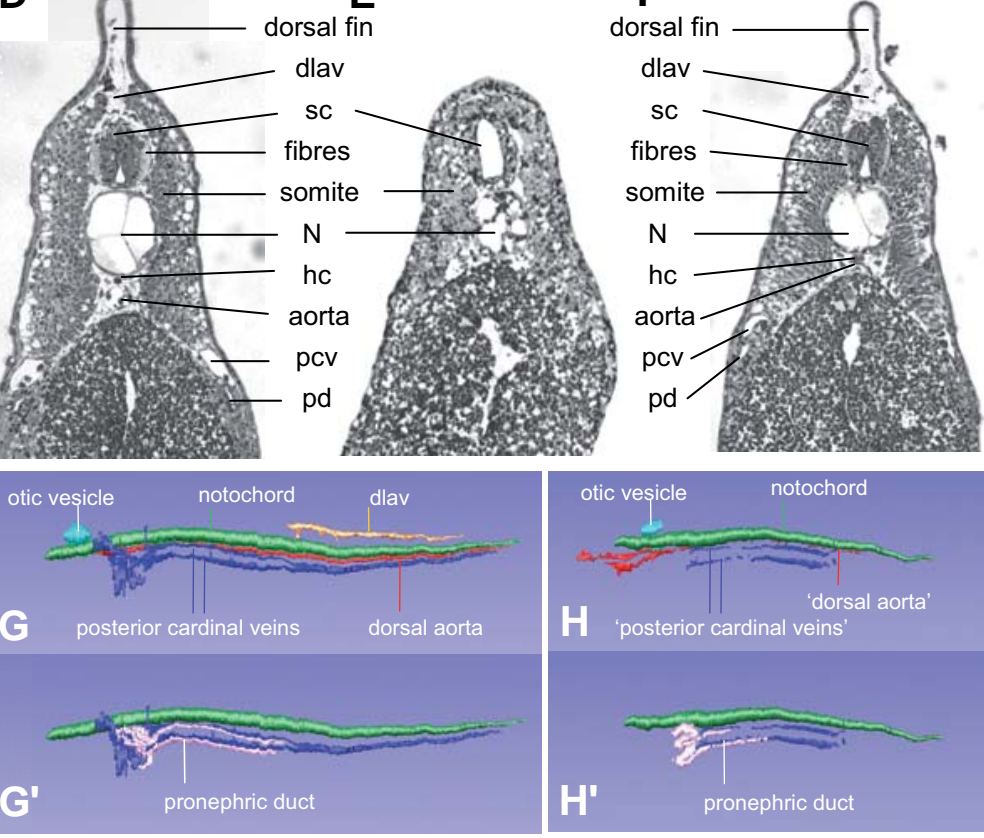

Fig. 2. Lef1 depletion results in impaired development of different tissues. Non-injected control X. tropicalis embryo, stage 38 (A). Lef1 morpholino oligo injected embryos (B) are short and lack a tail with a dorsal and ventral fin. The eyes are small and pigmentation is absent. Co-injection of mRNA encoding human LEF1 rescues the lef1 MO effects $(\mathbf{C})$. Broken lines in $(A-C)$ indicate level of sections shown in (D-F). Transverse section of non-injected control embryo (D). Lef1 MO injected embryos (E) lack the dorsal longitudinal anastomosing vessel (dlav), the posterior cardinal veins (pcv), the pronephric ducts ( $p d$ ), the hypochord (hc) and the dorsal aorta. The somites and notochord (N) are poorly differentiated. The spinal cord (sc) lacks nerve fibers and the notochord $(N)$ is smaller in diameter. Embryo co-injected with LEF1 RNA (F) shows rescue of the structures affected by lef1 depletion. 3D reconstructions of transverse sections ( $\left.\mathbf{G}-\mathbf{H}^{\prime}\right)$ of embryos shown in $(A, B)$. Noninjected control embryo (G, $\left.\mathbf{G}^{\prime}\right)$ and embryo injected with lef1 $M O\left(\mathbf{H}, \mathbf{H}^{\prime}\right)$. Reconstructions were aligned relative to the otic vesicle.

somites and gut, demonstrating that general patterning along the anterior-posterior and dorsal-ventral body axes was unaffected (Fig. 2B,E,H,H') compared to noninjected embryos (Fig. 2G,G'). However, multiple tissues and organs were abnormal as will be described below. Co-injection of human $\angle E F 1 \mathrm{mRNA}$ resulted in $82 \%(n=219 / 267)$ embryos with normal head, body, tail and fins (Fig. 2F). Rescued embryos also showed wild type melanophore patterns, reacted to mechanical stimuli and their internal organs were normal, demonstrating the specificity of the effects of the lef1 morpholino oligonucleotide.

\section{Lef1 depleted embryos show developmental defects in de- rivatives of the ectoderm}

At tail bud stages the patterning of the CNS of lef1 depleted embryos did not show gross abnormalities since expression of the pan-neural marker gene ncam (Kintner and Melton, 1987) (not shown) and of pax2 (Fig. 4F) in the CNS was not altered. However, histological analysis showed that only very few nerve 

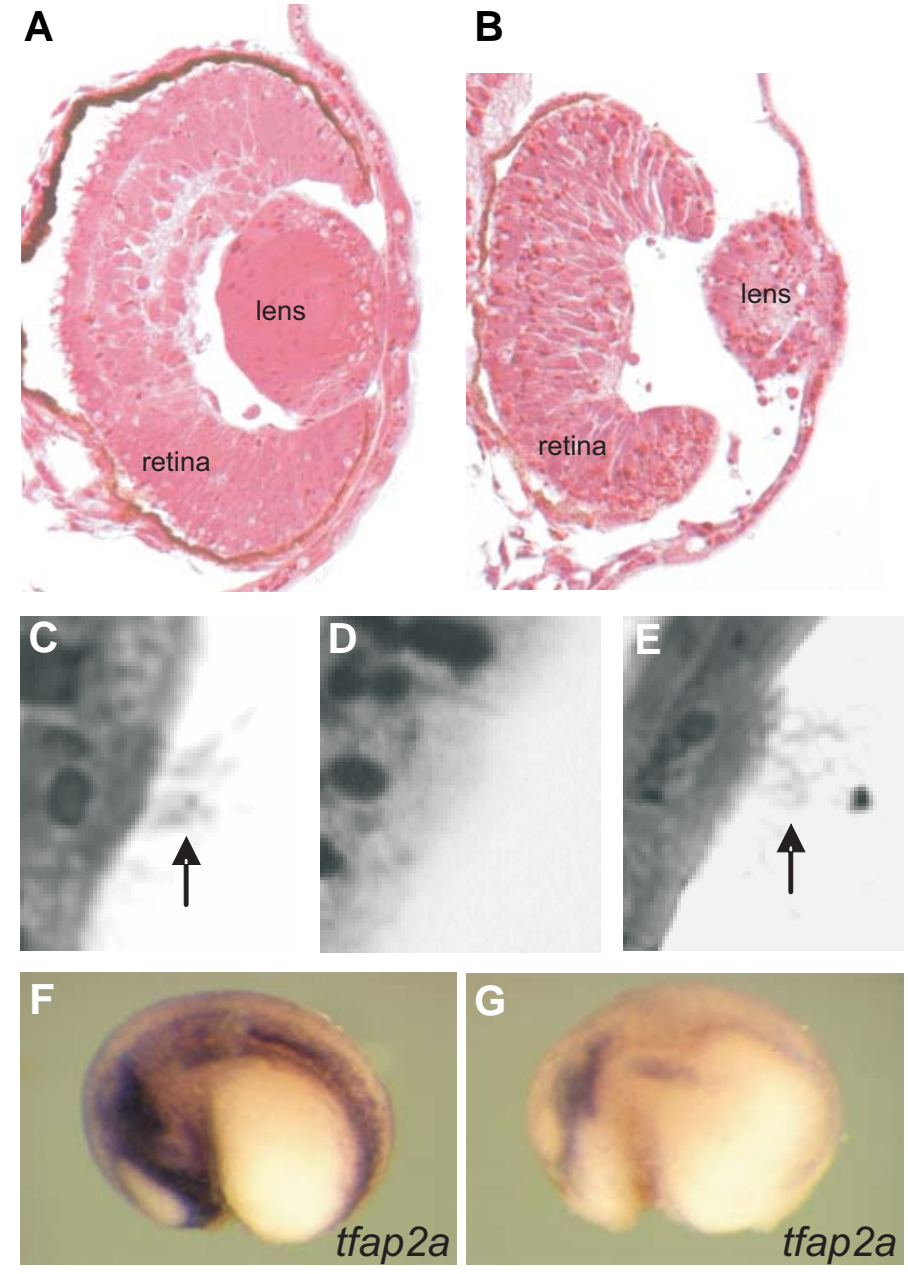

Fig. 3. Ectodermal derivatives are affected by lef1 depletion. Transverse section of the eye of a control X. tropicalis stage 38 embryo (A) shows differentiation of retina and lens. Transverse section of the eye of a lef1 depleted embryo (B) shows that the retina and lens have not developed properly and that the lens has not separated from the epidermal ectoderm. Detail of the epidermis showing a ciliated cell (arrow) of a control embryo (C). Lef1 depleted embryos (D) lack ciliated cells in the epidermis. Rescue of ciliated cells (arrow) in the epidermis of embryos injected with lef1 MO together with human LEF1 RNA (E). Expression of the neural crest marker gene tfap2a in a control embryo (F) and reduced expression in a lef1 depleted embryo (G).

fibers were present in the CNS at stage 38 (Fig. 2E) and that nerve fibers where absent in the eyes Fig. 3B). The eyes were smaller and less pigmented compared to those of non-injected controls (Figs $3 A, B$ ), while the eye lens remained connected to the epidermis (Fig. 3B), indicating a problem in tissue separation.

The epidermis of lef1 depleted embryos lacked ciliated cells, which are derived from the sensory layer of the ectoderm (Chu and Klymkowsky, 1989) (Fig. 3D). In addition, the epidermis was not well separated from the underlying somites (Fig. 2E), again indicating that tissue separation during early development was abnormal. Several ectodermal placodes, e.g. those for the pituitary and the cephalic ganglia of the sensory system were also affected in lef1 depleted embryos. The pituitary was very small compared to that of control embryos and neural crest derived cephalic ganglia were absent or very small (not shown). Also, in the lef1 mutant mouse, the ganglia of the trigeminal nerve (NV) were found to be absent (van Genderen et al., 1994). Thus, the sensory system may not be functioning properly in lef1 depleted embryos resulting in the observed lack of response to mechanical stimuli.

The endogenous expression pattern of Xenopus lef1 shows strong expression in the neural crest (NC) and the branchial arches (Molenaar et al., 1998) suggesting a role for lef1 during development of these tissues. Lef1 depleted embryos lack NC derived pigmented cells (Fig. 2B), indicating a defect in melanophore generation and/or differentiation. The heads of lef1 depleted embryos were small presumably because of disturbed development of neural crest derived mesenchyme. Moreover, the expression of the NC marker gene tfap2a (Luo et al., 2003)was strongly reduced in lef1 depleted embryos (Fig. 3G) indicating that lef1 plays a role in neural crest differentiation.

\section{Axial and paraxial mesoderm differentiation is affected in lef1 depleted embryos}

Tissues derived from the axial and paraxial mesoderm were also affected in lef1 depleted embryos. The notochord was smaller in diameter and length. The notochordal cells have smaller vacuoles than do wild-type notochords (Figs 2D,E) and did not show the typical epithelial configuration as in wild type (Fig. 2D) and rescued (Fig. 2F) embryos. Signals from the notochord are necessary for the development of the hypochord, which is derived from the endoderm (Cleaver et al., 2000). In lef1 depleted embryos the hypochord was absent (Fig. 2E), suggesting that signaling from the notochord was impaired.

We previously showed that lef1 is required for myod expression already during gastrulation (Roël et al., 2002). Therefore we further analysed the formation of the somites and myod expression also later in development, at tadpole stages. The ventral parts of the somites of lef 1 depleted embryos did not show distinct intersomitic boundaries (Fig. 4B) and lacked myod expression (Fig. 4D) compared to control embryos (Fig. 4 A,C). Moreover, the ventral parts of the somites did not form hypaxial musculature (Figs 4D), which normally migrates from the somites to form the body wall musculature in control embryos (Fig. 4C; Martin and Harland, 2001). In the posterior part of the embryo the tissue boundaries between the somites and the epidermis and between the somites and the neural tube were also not distinct (Fig. 2E). These results again indicate a role for lef1 in tissue separation.

\section{Derivatives of the lateral plate mesoderm are absent or abnormal in lef1 depleted embryos}

The dorsal part of the lateral plate mesoderm (DLP) gives rise to cells that will eventually form the pronephric ducts and some of the major blood vessels (Walmsley et al., 2002). Lef1 is expressed in the lateral plate mesoderm both in Xenopus and the mouse (Molenaar et al., 1998; Galceran et al., 1999). In lef1 depleted embryos the pronephric ducts were absent or incomplete (Figs 2E, $\mathrm{H}^{\prime}$ ). Expression of pax2, a marker for the pronephric tubules and pronephric duct (Heller and Brändli, 1997; Drawbridge et al., 2003) was absent posteriorly (Fig. 4F). Also, more anterior pax2expressing cells did not properly develop into pronephric tubules and proximal pronephric ducts (Figs $2 \mathrm{H}^{\prime}$ and $4 \mathrm{G}, \mathrm{H})$. Co-injection of human $\angle E F 1 \mathrm{RNA}$ rescued these effects 
(Fig. 2F). These results indicate a role for lef1 in patterning the DLP mesoderm.

\section{Development of the major blood vessels and the heart is affected by lef1 depletion}

The lack of blood flow in stage 38 embryos, as well as the abolished expression of the endothelial marker msr during gastrulation lead us to further investigate the formation of the vascular system in lef1 deficient embryos. Histological analysis revealed impaired formation of the dorsal aorta and the posterior cardinal veins (pcv) (Figs. 2E,H) and absence of the dorsal longitudinal anastomosing vessel (dlav) (Figs. 2E, H, 5B) when compared with control embryos of the same stage (Fig. 2D,G, $5 A)$. The formation of these structures was rescued by coinjection of human $\angle E F 1$ RNA (Fig. 2F). We analyzed the expression of the endothelial marker genes flit (Meyer et al., 1995) and msr (Devic et al., 1996), the homologue of the human apelin receptor APJ (O'Dowd et al., 1993). Fli1 and msr/APJ are expressed in overlapping domains in the endothelial precursor cells of the DLP mesoderm (Walmsley et al., 2002) and subsequently in the dorsal aorta, pcv, intersomitic vessels and dlav (Fig. 5C,E and Meyer et al., 1995; Devic et al., 1996). Expression of fli1 and $m s r / A P J$ in the prospective dorsal aorta and pcv was strongly decreased (Figs. 5D,F) and the formation of endothelial tubules was blocked in lef1 depleted embryos (Figs 2E,H, 5B). Endothelial precursor cells of the DLP that will form the pcv develop in close proximity to the ventral parts of the somites where angiogenic sprouting of the pcv gives rise to formation of intersomitic vessels (Helbling et al., 2000). Fli1 and msr/APJexpressing cells were also absent between somites (isv, intersomitic vessels) in lef1 depleted embryos (Figs. 5D,F), most likely as a secondary effect of the abnormal formation of the pcv.

As mentioned before, the formation of the hypochord is impaired in lef1 depleted embryos (Fig. 2E). Because the hypochord is required for proper formation of the aorta (Cleaver and Krieg, 1998; Cleaver et al., 2000) our results suggest an indirect effect of lef 1 depletion on blood vessel formation in addition to a direct function in vasculogenesis.

Since Xenopus lef1 is expressed in the presumptive endocar- dium (Molenaar et al., 1998; Fig. 5G), which also develops by vasculogenic mechanisms (Coffin and Poole, 1991), we examined whether endocardium formation was affected in lef1 depleted embryos. Analysis of the heart of stage 38 lef1 depleted embryos showed severe retardation of the development of both the endocardium and the myocardium (Fig. 5I). Expression of the endothelial marker gene $m s r$, which is normally expressed in the endocardium and ventral aorta (Devic et al., 1996), was abolished in the heart of lef1 depleted embryos (Fig. 5F).

Our results indicate that lef1 dependent pathways play an important role in expression of the endothelial-associated gene msr/APJ during gastrulation as well as during blood vessel formation and heart development.

\section{Discussion}

Our experiments reveal that knockdown of lef1 leads to early developmental defects in the derivatives of the mesoderm and ectoderm in Xenopus, as expected from the expression pattern of lef1. In the mouse, targeted inactivation of Lef1, or Tcf1, did not produce early phenotypes, only null mutations in both Lef1 and Tcf1 caused a severe defect in the differentiation of paraxial mesoderm at the same time leading to the formation of additional neural tubes (Galceran et al., 1999). A redundant role of Lef1 and Tcf1 in Wnt signaling during early mouse development explained these results (Galceran et al., 1999). Since tcf1 is also expressed during early development in Xenopus (Roël et al., 2003), redundancy between lef1 and tcf1 functions is less or absent during Xenopus development. This difference may relate to the differences in the timing of paraxial mesoderm differentiation between mouse and Xenopus (Pownall et al., 2002). Also, differences in the expression of Lef1 iso-forms between mouse and Xenopus may be important. Human $\angle E F 1$ exon $\mathrm{VI}$ is naturally differentially spliced (Arce et al., 2006). Furthermore, natural dominant negative LEF1 is present in normal human and murine thymus tissue (Arce et al., 2006; Travis et al., 1991). We showed that the genomic sequences of Xenopus and Fugu lef1 do not contain exon VI. Only a LEF1 isoform containing exon VI (Arce et al., 2006) can efficiently induce formation of an ectopic axis and

Fig. 4. Mesoderm formation is affected by lef1 depletion. Somitic boundaries of a control X. tropicalis embryo (A). The intersomitic boundaries (white arrow heads) are affected by lef1 depletion (B). At the ventral side boundaries are lost. Myod expression in a control $\mathrm{X}$. tropicalis stage 38 embryo $(\mathbf{C})$ in the ventral part of somites (black arrowhead) and migrating hypaxial musculature (open arrowheads). Myod expression is downregulated in the ventral part of somites (black arrow-
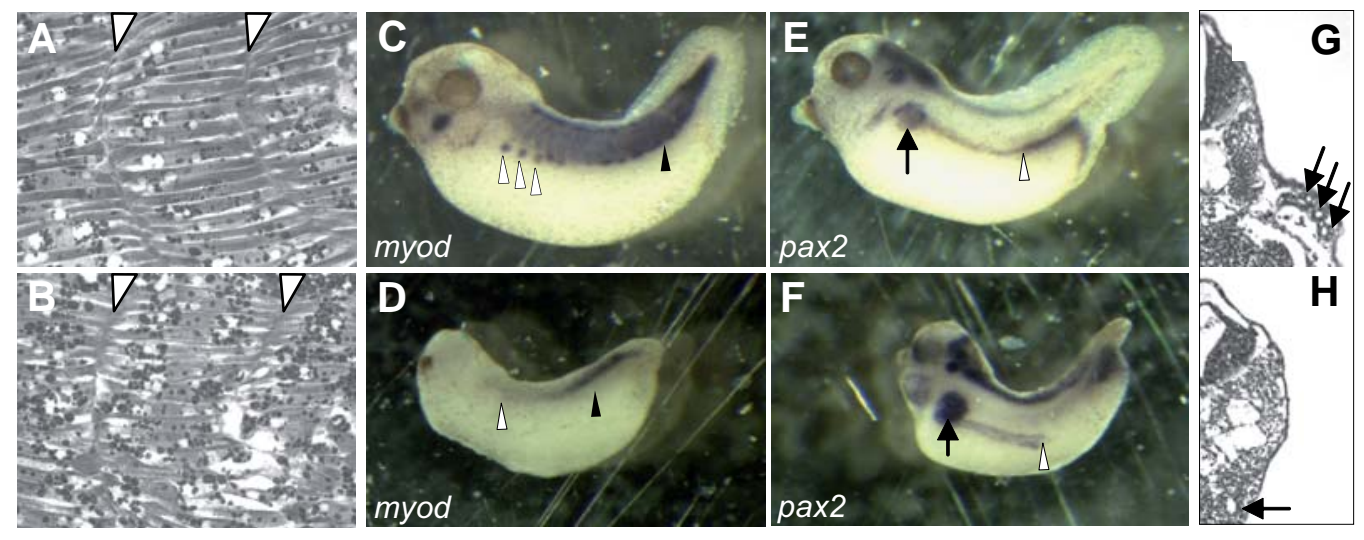

head) and hypaxial musculature is absent in lef1 depleted embryos (D). Control embryos stained for pax2 expression (E) in the pronephros (black arrow), pronephric duct (open arrowhead indicates junction between pronephric duct and rectal diverticulum) and rectal diverticulum. In lef1 depleted embryos (F) the caudal part of the pronephric ducts did not grow out properly and did not join the rectal diverticula, which were rudimentary (open arrowhead indicates the posterior end of an aberrant pronephric duct). Transverse section of the pronephros (G) showing pronephric tubules in a control embryo (arrows). The pronephros of lef1 depleted embryos (H) remains a single tube (arrow), with an S-shape (cf. Fig. 4F). 
enhance siamois expression (Gradl et al., 2002). Our rescue experiments in which the human LEF1 isoform was used lacking the activation domain encoded by exon $\mathrm{VI}$ demonstrate that this domain is not important for activation of downstream targets, like myod(Roël et al., 2002) and msr/APJ (this study) in then Xenopus embryo. The $\beta$-catenin binding domain on the other hand is essential for lef 1 signaling since ectopic expression of a lef1 construct lacking the $\beta$-catenin BD abolishes myod expression (Roël et al., 2002).

The phenotype of lef1 knockdown embryos as described here, is different from that obtained after depletion of tcf3 (Houston et al., 2002) confirming non-redundant functions of these proteins during early development of Xenopus (Roël et al., 2002).

\section{Lef1 is required to pattern the ventral and dorsal mesoderm}

We show that lef1 is required for the proper expression of myod, msr/APJand nodal related 3 in the ventral and dorsal mesoderm. Myod(Roël et al., 2002) but not myf5 expression (this study) requires lef1 in the mesodermal myotomal progenitors in the paraxial mesoderm during gastrulation. Furthermore, our results indicate that lef1 is not only required for expression of myod during gastrulation in the earliest myotomal progenitors but also in their hypaxial migratory derivatives during body wall formation. We noticed that the ventral parts of the somites showed abnormal intersomitic boundaries and that somites were not distinctly separated from surrounding tissues. The latter results suggest that lef1 is required in the (pre)somitic mesoderm, as in other locations (see below), for proper tissue separation and/ or boundary formation.

In mouse paraxial mesoderm explants both Myod and Myf5 are Wnt responsive (Tajbakhsh et al., 1998) and several studies have suggested that myf5expression may be regulated by $\beta$-catenin/tcf in Xenopus (Yang et al., 2002; Shi et al., 2002). Although depletion of lef 1 in Xenopus does not lead to inhibition of myf5 expression, we noticed that co-injection of human $\angle E F 1$ RNA without exon VI sequences leads to ectopic myf5 expression across the dorsal midline (DM). In the DM myf5expression may be repressed by Tcf factors (Yang et al., 2002), whereas myod expression requires an activator function of lef1 (Roël et al., 2002). Indeed, knockdown of tcf3 leads to ectopic expression of myf5 across the dorsal midline at early gastrula stages (G. Roël, unpublished). These results indicate different functions for lef1 and tcf3 in the regulation of myod and myf5 expression.

We observed ectopic expression of nodal related 3 in the dorsal mesoderm upon lef1 depletion. This suggests a repressor function for lef1, which would be in line with results obtained in cell lines (Brantjes et al., 2001). Although nodal related 3 is thought to be directly regulated by Wnt/Tcf (McKendry et al., 1997) the observed effects in lef1 depleted embryos could also be indirect. Thus, whether the observed effects of lef1 knockdown on the expression of myod, msr/APJor nodal related 3 in the ventral and
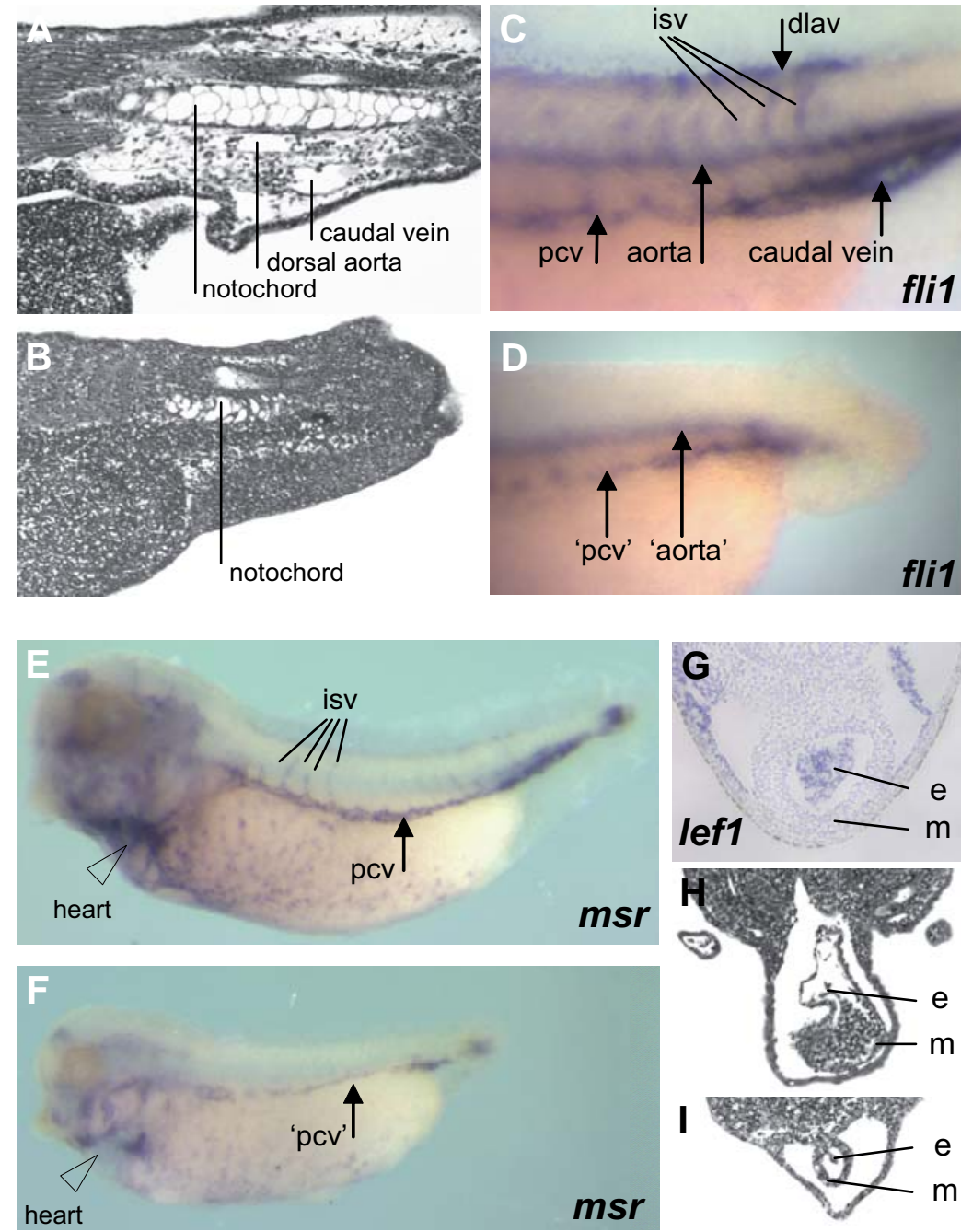

Fig. 5. The cardiovascular system is affected by lef1 depletion. Sagittal section of a stage 38 control embryo (A) showing the dorsal aorta and the caudal vein. In lef1 depleted embryos (B), the tail lacks the major blood vessels, the caudal vein and the dorsal aorta. Fli1 expression in the endothelium of a control embryo (C). isv, intersomitic vessels. dlav, dorsal longitudinal anastomosing vessel. pcv, posterior cardinal vein. aorta, dorsal aorta. Lef1 depleted embryos show strongly reduced expression of fli1 (D) at the position of the pcV and the aorta and no indication of intersomitic vessels, dlav or caudal vein. Msr/APJ expression in control embryo (E) and in lef1 depleted embryo (F) showing reduced expression. Endogenous expression of lef1 in the heart of a stage 34 control embryo (G). e, endocardium. $m$, myocardium. Transverse section of the heart of a control embryo $\mathbf{( H )}$ and of a lef1 depleted embryo (I) which shows severe retardation of heart development both for the endocardium and myocardium.

dorsal mesoderm are direct or indirect remains to be established.

\section{Lef1 functions in the neural crest}

The endogenous expression of lef1 in neural crest cells (Molenaar et al., 1998) indicates a function in the differentiation of these cells. Indeed, lef1 depleted embryos showed developmental defects in several derivatives of the neural crest like melanocytes and sensory ganglia. Formation and expansion of the neural crest depends on Wnt signals (Yanfeng et al., 2003). In Wnt1/ Wnt3a double mutant mice tfap2a expression was down regu- 
lated (Ikeya et al., 1997) as we also observed in lef1 depleted $X$. tropicalis embryos. Tfap2a expression was recently found to be responsive to Wnt signals (Luo et al., 2003) and its expression may be directly regulated by lef1 in the neural crest. However, we cannot discriminate between direct effects of lef1 depletion in the neural crest cells and indirect effects e.g. through the paraxial mesoderm (Monsoro-Burq et al., 2003).

\section{A role for Xenopus lef1 in cell adhesion}

An iterative feature in lef1 knockdown embryos that we observed seems to be that different cell types derived from different germ layers are not able to separate from their initial context. Examples are neural crest cells, which need to delaminate before they can migrate (Yanfeng et al., 2003); the eye lens, which buds of and separates from the surface ectoderm (Grimes et al., 1998); ciliated cells of the epidermis, which migrate from the inner, sensorial layer into the outer layer (Deblandre et al., 1999); hypaxial muscle precursors, which leave the somites and migrate into the ventral body wall (Martin and Harland, 2001); the hypochord which separates from the endoderm (Cleaver et al., 2000) and dorsal aorta precursor cells, which need to escape from the lateral plate mesoderm before they can migrate towards the hypochord (Cleaver and Krieg, 1998). In these processes lef1 may play a role in the regulation of differential cell-cell adhesion.

\section{Xenopus lef1 is required for development of the cardiovascu- lar system}

We showed that lef1 depletion results in the impaired development of major blood vessels and the heart. The dorsal aorta and the posterior cardinal veins arise from cells of the dorsal side of the lateral plate mesoderm (Walmsley et al., 2002) by different cellular processes. The precursor cells of the future dorsal aorta require signals from the hypochord to migrate from the lateral plate mesoderm towards the hypochord (Cleaver and Krieg, 1998; Cleaver et al., 2000). The lack of the hypochord in lef1 depleted embryos may be causative for the absence of the dorsal aorta but not for the absence of the posterior cardinal veins which are normally formed directly from the lateral plate mesoderm. Since lef1, fli1 and $m s r / A P J$ are co-expressed in the lateral plate mesoderm and since $m s r / A P J$ expression requires lef1, our data suggest that lef1 functions in the specification of angioblasts when they still reside in the lateral plate mesoderm. In addition to impaired cell fate specification, the effects of lef1 depletion on blood vessel development may be caused by blocking the separation and subsequent expansion of the number of precursor cells in the lateral plate mesoderm.

From results of endoderm-depleted embryos it was concluded that signals for angioblast specification are coming from the mesoderm, which is in line with our data, and that signals required for endothelial tube formation come from the endoderm (Vokes and Krieg, 2002). These signals are presently unknown except for the VEGF excreting hypochord, which is an endoderm derivative and which is absent in lef 1 depleted embryos. This may explain why endothelial tubes were not formed in lef1 depleted embryos while still some flit and msr/APJ positive precursor cells were present. Another indirect effect on vasculogenesis in the lef1 depleted embryos may be caused by the somites that did not differentiate correctly and therefore may not function properly as a signaling compartment to induce blood vessel formation (Cleaver and Krieg, 1998). Because development of the posterior cardinal vein is impaired in lef1 depleted embryos we think that the lack of angiogenic sprouting of the intersomitic vessels in lef1 depleted embryos is secondary to the effects of lef 1 depletion on vasculogenesis possibly in combination with impaired signaling from the affected somites (Rossant and Howard, 2002).

The early steps in cardiogenesis are believed to be regulated by activation of the $\mathrm{Wnt} / \mathrm{Ca}^{2+}$ pathway as well as both activation and repression of the $\mathrm{Wnt} / \beta$-catenin pathway, during different stages of heart development (reviewed in Eisenberg and Eisenberg, 2006). In addition, recent studies show that msr/APJ and its ligand apelin are both required for proper development of the heart and blood vessels during Xenopus development (Inui et al., 2006). This is in line with our results showing that lef1 is required for the expression of $m s r / A P J$ during gastrulation and also for the development of the endocardium and myocardium during later stages.

\section{Materials and Methods}

\section{Analysis of Xenopus and Fugu lef1 genomic sequences}

$X$. laevis genomic DNA was purified by standard methods (Davis et al., 1986). Primers used: exon V fwd: 5'-GGCCAGATGACACCACCATTGG, exon VII: 5'-CCCGGAGGACCAGAAACCATATG. A genomic DNA fragment was amplified using the Advantage PCR kit (Clontech) and ligated into the pGEM-T easy vector (Promega) and sequenced using a 373 DNA sequencer (Perkin Elmer). Fugu genomic sequences were derived from the Fugu rubripes database of the DOE Joint Genome Institute (www.jgi.doe.gov).

\section{RNA synthesis for capped mRNA and RNA antisense probes}

Capped RNA encoding the human LEF1 isoform without exon VI (van de Wetering etal., 1996) was synthesized using mMessage mMachine T7 (Ambion). For synthesis of DIG labeled (Roche) antisense RNA probes plasmid DNAs were linearized and used as template. RNA was purified using RNeasy columns (QIAGEN). Plasmid DNAs were kindly provided by A. Brändli (Xenopus pax2), M. Baltzinger (Xenopus fli1), S. Hoppler (Xenopus wnt8), C. Kintner (Xenopus ncam) and J. Smith (Xenopus brachyury). X. tropicalis specific probes, lef1, myod, msr/APJ and tfap2a, were constructed by RT-PCR using primer sequences designed from the $X$. tropicalis EST database (Sanger Center). X. tropicalis myf5 probe was generated according to Polli and Amaya (2002). PCR fragments for probes were ligated into the pGEM-T easy vector (Promega).

\section{Embryo manipulation}

$X$. tropicalisembryos were obtained by in vivofertilization and injected with $20 \mathrm{ng}$ lef1 morpholino antisense oligonucleotide (Gene Tools LLC, Roël et al., 2002), which is complementary to the translation start site of $X$. tropicalis lef1 or $20 \mathrm{ng}$ lef1 $\mathrm{MO}$ together with $180 \mathrm{pg}$ RNA encoding human LEF1 to restore Lef1 protein levels in the embryo. Human LEF1 isoform without exon VI was used (van de Wetering et al., 1996). The human LEF1 RNA is not sensitive to the lef1 MO since its sequence contains 6 mismatches. Embryos were injected in both blastomeres at the 2 -cell stage and raised at $23^{\circ} \mathrm{C}$ in $12 \%$ MMR (Peng et al., 1991). Developmental stages of Xenopus embryos were determined according to Nieuwkoop and Faber (1967). Embryos were fixed in MEMPFA (100 $\mathrm{mM}$ MOPS, pH 7.4, 2 mM EGTA, 1mM MgSO4, 4\% paraformaldehyde) for whole-mount in situ hybridization and sectioning.

\section{Whole mount in situ hybridization, sectioning and 3D-reconstruc- tions}

Whole mount in situhybridization was performed as described before (Molenaar et al., 1998) with modifications for $X$. tropicalis: hybridization at 
$65^{\circ} \mathrm{C}$ without $\mathrm{RNase}$ treatment for all probes except $X$. tropicalis myf5 $\left(65^{\circ} \mathrm{C}\right.$, with RNase treatment). Embryos were embedded in Technovit 8100 and sectioned at $7 \mu \mathrm{m}$ for histological analysis. Serial sections of transversally sectioned embryos were used as input for the TDR-3dbase (Verbeek, J. et al., 1995), which reconstructs serial sections into a 3 dimensional image.

\section{Acknowledgments}

We thank A. Brändli, M. Baltzinger, S. Hoppler, C. Kintner and J. Smith for plasmids. We thank $N$. Spieker for critical reading of the manuscript and members of the lab for helpful discussions. We thank J. Korving for technical assistance. This work was supported by the Earth and Life Sciences Foundation ALW, subsidized by the Netherlands Organization for Scientific Research NWO and the EU (QLRT-2000-01275).

\section{References}

ARCE, L., YOKOYAMA, N.N. and WATERMAN, M.L. (2006). Diversity of LEF/TCF action in development and disease. Oncogene 25: 7492-7504.

BRANTJES, H., ROOSE, J., VAN DE WETERING, M. and CLEVERS, H. (2001). All TCF HMG box transcription factors interact with Groucho-related corepressors. Nucleic Acids Res. 29: 1410-1419.

CHRISTIAN, J.L. and MOON, R.T. (1993). Interactions between Xwnt-8 and Spemann organizer signaling pathways generate dorsoventral pattern in the embryonic mesoderm of Xenopus. Genes Dev. 7: 13-28.

CHU, D.T. and KLYMKOWSKY, M.W. (1989). The appearance of acetylated alphatubulin during early development and cellular differentiation in Xenopus. Dev. Biol. 136: 104-117.

CLEAVER, O. and KRIEG, P.A. (1998). VEGF mediates angioblast migration during development of the dorsal aorta in Xenopus. Development 125: 39053914.

CLEAVER, O., SEUFERT, D.W. and KRIEG, P.A. (2000). Endoderm patterning by the notochord: development of the hypochord in Xenopus. Development 127: 869-879.

COFFIN, J.D. and POOLE, T.J. (1991). Endothelial cell origin and migration in embryonic heart and cranial blood vessel development. Anat. Rec. 231: 383395.

DAVIS, L.G., DIBNER, M. and BATTEY, J. (1986). Basic Methods in Molecular Biology. Elsevier Science Publishing Co., Inc. New York. pp. 44-46.

DEBLANDRE, G.A., WETTSTEIN, D.A., KOYANO-NAKAGAWA, N. and KINTNER, C. (1999). A two-step mechanism generates the spacing pattern of the ciliated cells in the skin of Xenopus embryos. Development 126: 4715-4728.

DEVIC, E., PAQUEREAU, L., VERNIER, P., KNIBIEHLER, B. and AUDIGIER, Y (1996). Expression of a new $G$ protein-coupled receptor Xmsr is associated with an endothelial lineage in Xenopus laevis. Mech. Dev. 59: 129-140.

DRAWBRIDGE, J., MEIGHAN, C.M., LUMPKINS, R. and KITE, M.E. (2003). Pronephric duct extension in amphibian embryos: Migration and other mechanisms. Dev. Dyn. 226: 1-11.

EISENBERG, L.M. and EISENBERG, C.A. (2006). Wnt signal transduction and the formation of the myocardium. Dev. Biol. 293: 305-315.

GALCERAN, J., FARIÑAS, I., DEPEW, M.J., CLEVERS, H. and GROSSCHEDL, R. (1999). Wnt3a-/- -like phenotype and limb deficiency in Lef1-/-Tcf1-/- mice. Genes Dev. 13: 709-717.

GRADL, D., KONIG, A. and WEDLICH, D. (2002). Functional diversity of Xenopus lymphoid enhancer factor/T-cell factor transcription factors relies on combinations of activating and repressing elements. J. Biol. Chem. 277: 14159-14171.

GRIMES, P.A., KOEBERLEIN, B., FAVOR, J., NEUHAUSER-KLAUS, A. and STAMBOLIAN D. (1998). Abnormal eye development associated with Cat4a, a dominant mouse cataract mutation on chromosome 8. Invest. Ophthalmol. Vis. Sci. 39: 1863-1869.

HELBLING, P.M., SAULNIER, D.M. and BRÄNDLI, A.W. (2000). The receptor tyrosine kinase EphB4 and ephrin-B ligands restrict angiogenic growth of embryonic veins in Xenopus laevis. Development 127: 269-278.

HELLER, N. and BRÄNDLI, A.W. (1997). Xenopus Pax-2 displays multiple splice forms during embryogenesis and pronephric kidney development. Mech. Dev.
69: 83-104.

HOPPLER, S., BROWN, J.D. and MOON, R.T. (1996). Expression of a dominantnegative Wnt blocks induction of MyoD in Xenopus embryos. Genes Dev. 10 2805-2817.

HOPPLER, S. and MOON, R.T. (1998). BMP-2/-4 and Wnt-8 cooperatively pattern the Xenopus mesoderm. Mech. Dev. 71: 119-129.

HOUSTON, D.W., KOFRON, M., RESNIK, E., LANGLAND, R., DESTRÉE, O. WYLIE. C. and HEASMAN, J. (2002). Repression of organizer genes in dorsal and ventral Xenopus cells mediated by maternal XTcf3. Development 129: 4015-4025.

IKEYA, M., LEE, S.M., JOHNSON, J.E., MCMAHON, A.P. and TAKADA, S. (1997). Wnt signalling required for expansion of neural crest and CNS progenitors. Nature 389: 966-970.

INUI, M., FUKUI, A., ITO, Y. and ASASHIMA, M. (2006). Xapelin and Xmsr are required for cardiovascular development in Xenopus laevis. Dev. Biol. 298:188 200.

KINTNER, C.R. and MELTON, D.A. (1987). Expression of Xenopus N-CAM RNA in ectoderm is an early response to neural induction. Development99:311-325.

LUO, T., LEE, Y.H., SAINT-JEANNET, J.P. and SARGENT, T.D. (2003). Induction of neural crest in Xenopus by transcription factor AP2alpha. Proc Nat/ Acad Sci USA. 100: 532-537.

MARTIN, B.L. and HARLAND, R.M. (2001). Hypaxial muscle migration during primary myogenesis in Xenopus laevis. Dev. Biol. 239: 270-280.

MCKENDRY, R., HSU, S.C., HARLAND, R.M. and GROSSCHEDL, R. (1997). LEF$1 /$ TCF proteins mediate wnt-inducible transcription from the Xenopus nodalrelated 3 promoter. Dev. Biol. 192: 420-431.

MEYER, D., STIEGLER, P. HINDELANG, C., MAGER, A.M. and REMY, P. (1995). Whole-mount in situ hybridization reveals the expression of the XI-Fli gene in several lineages of migrating cells in Xenopus embryos. Int. J. Dev. Biol. 39 : 909-919.

MOLENAAR, M., ROOSE, J., PETERSON, J., VENANZI, S., CLEVERS, H. and DESTRÉE, O. (1998). Differential expression of the HMG box transcription factors XTcf3 and XLef1 during early Xenopus development. Mech. Dev. 75 $151-154$

MONSORO-BURQ, A.H., FLETCHER, R.H. and HARLAND, R.M. (2003). Neural crest induction by paraxial mesoderm in Xenopus embryos requires FGF signals. Development 130: 3111-3124.

NIEUWKOOP, P.D. and FABER, J. (1967). Normal table of Xenopus laevis (Daudin). Amsterdam: North Holland Publishing Company.

NUTT, S.L., BRONCHAIN, O.J., HARTLEY, K.O. and AMAYA, E. (2001). Comparison of morpholino based translational inhibition during the development of Xenopus laevis and Xenopus tropicalis. Genesis 30: 110-113.

O'DOWD, B. F., HEIBER, M., CHAN, A., HENG, H.H., TSUI, L.C., KENNEDY, J.L., SHI, X., PETRONIS, A., GEORGE, S.R. and NGUYEN, T. (1993). A human gene that shows identity with the gene encoding the angiotensin receptor is located on chromosome 11. Gene 136: 355-360.

OOSTERWEGEL, M., VAN DE WETERING, M., TIMMERMAN, J., KRUISBEEK, A., DESTRÉE, O., MEIJLINK, F. and CLEVERS, H. (1993). Differential expression of the HMG box factors TCF-1 and LEF-1 during murine embryogenesis. Development 118: 439-448.

PENG, H.B. (1991). In Kay, B.K. and Peng, H.B. (eds.), Appendix A: Solutions and Protocols, vol. 36. Academic Press, Inc., San Diego, California, pp. 675-662.

POLLI, M. and AMAYA, E. (2002). A study of mesoderm patterning through the analysis of the regulation of Xmyf-5 expression. Development 129: 2917-2927.

POWNALL, M.E., GUSTAFSSON, M.K. and EMERSON, C.P. (2002). Myogenic regulatory factors and the specification of muscle progenitors in vertebrate embryos. Annu. Rev. Cell Dev. Biol. 18: 747-783.

ROËL, G., HAMILTON, F., GENT, Y., BAIN, A., DESTRÉE, O. and HOPPLER, S. (2002). Lef-1 and Tcf-3 transcription factors mediate tissue-specific Wnt signalling during Xenopus development. Curr. Biol. 12: 1941-1945.

ROËL, G., VAN DEN BROEK, O., SPIEKER, N., PETERSON-MADURO, J. and DESTRÉE, O. (2003). Tcf-1 expression during Xenopus development. Gene Expr. Patterns 3: 123-126.

ROSSANT, J. and HOWARD, L. (2002). Signaling pathways in vascular development. Annu. Rev. Cell Dev. Biol.18: 541-573. 
SCHOHL, A. and FAGOTTO, F. (2002). Beta-catenin, MAPK and Smad signaling during early Xenopus development. Development 129: 37-52.

SHI, D.L., BOURDELAS, A., UMBHAUER, M. and BOUCAUT, J.C. (2002). Zygotic Wnt/ $\beta$-catenin signaling preferentially regulates the expression of Myf5 gene in the mesoderm of Xenopus. Dev. Biol. 245: 124-135.

TAJBAKHSH, S., BORELLO, U., VIVARELLI, E., KELLY, R., PAPKOFF, J., DUPREZ, D., BUCKINGHAM, M. and COSSU, G. (1998). Differential activation of Myf5 and MyoD by different Wnts in explants of mouse paraxial mesoderm and the later activation of myogenesis in the absence of Myf5. Development 125: 4155-4162.

TRAVIS, A., AMSTERDAM, A., BELANGER, C. and GROSSCHEDL, R. (1991). LEF-1, a gene encoding a lymphoid-specific protein with an HMG domain, regulates T-cell receptor alpha enhancer function. Genes Dev. 5: 880-894.

VAN DE WETERING, M., CASTROP, J., KORINEK, V. and CLEVERS, H. (1996). Extensive alternative splicing and dual promoter usage generate Tcf-1 protein isoforms with differential transcription control properties. Mol. Cel/ Biol. 16: 745752.

VAN GENDEREN, C., OKAMURA, R.M., FARINAS, I., QUO, R.G., PARSLOW, T.G., BRUHN, L. and GROSSCHEDL, R. (1994). Development of several organs that require inductive epithelial-mesenchymal interactions is impaired in LEF-1-deficient mice. Genes Dev. 8: 2691-2703.

VERBEEK, J. HUIJSMANS, D.P., BAETEN, R.J.A.M., SCHOUTSEN, N.J.C. and LAMERS, W.H. (1995). Design and implementation of a database and program for 3D reconstruction from serial sections: a data-driven approach. Microsc. Res. Tech. 30: 496-512.

VOKES, S.A. and KRIEG, P.A. (2002). Endoderm is required for vascular endothelial tube formation, but not for angioblast specification. Development 129: 775785.

WALMSLEY, M., CIAU-UITZ, A. and PATIENT, R. (2002). Adult and embryonic blood and endothelium derive from distinct precursor populations which are differentially programmed by BMP in Xenopus. Development 129: 5683-5695.

YANG, J., MEI, W., OTTO, A., XIAO, L., TAO, Q., GENG, X., RUPP, R.A. and DING, $X$. (2002). Repression through a distal TCF-3 binding site restricts Xenopus myf5 expression in gastrula mesoderm. Mech Dev. 115: 79-89.

YANFENG, W., SAINT-JEANNET, J.P. and KLEIN, P.S. (2003). Wnt-frizzled signaling in the induction and differentiation of the neural crest. Bioessays 25: 317-325.

\section{Further Related Reading, published previously in the Int. J. Dev. Biol.}

See our Special Issue The Spemann-Mangold Organizer edited by Edward de Robertis and Juan Aréchaga at: http://www.ijdb.ehu.es/web/contents.php?vol=45\&issue=1

See our recent Special Issue Fertilization, in honor of David L. Garbers and edited by Paul M. Wassarman and Victor D. Vacquier at: http://www.ijdb.ehu.es/web/contents.php?vol=52\&issue=5-6

Genetic interaction between Lef1 and Alx4 is required for early embryonic development

Kata Boras-Granic, Rudolf Grosschedl and Paul A. Hamel

Int. J. Dev. Biol. (2006) 50: 601-610
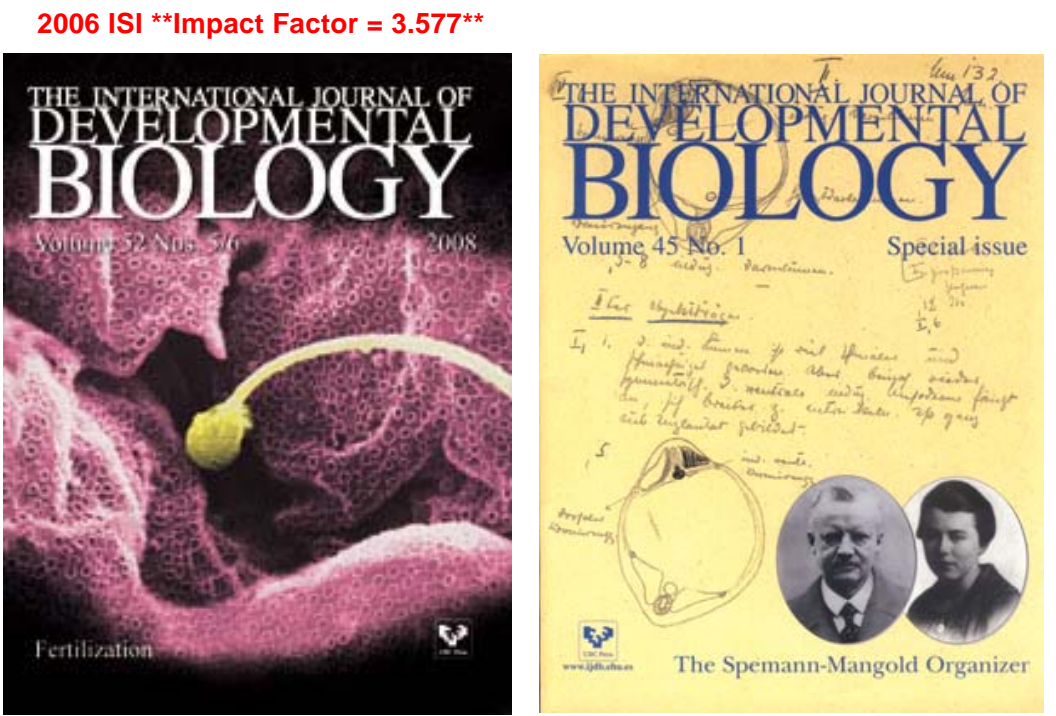